When we remember the effect of applying these agents to the mucous membrane lining the nostrils, the free secretion provoked lasting in many cases for twenty to forty hours, we can appreciate the amount of fluid thrown out from the uterine mucous membrane after applications of similar nature.

There is a class of cases having a profuse discharge from the endometrium, the characteristic glairy, white of egg mucus; some physicians treat these cases by merely placing a pledget of medicated cotton against the cervix uteri. This tampon effectually prevents all discharge from passing out of the uterine canal, the cavity becomes distended, the purulent secretion may pass into the Fallopian tubes, setting up a similar inflammatory condition in their mucous membrane as already exists in the walls of the uterus. The tampon under these conditions is dangerous, is productive of great mischief applied in this manner. Many a woman has had gonorthoal inflammation forced from the vagina and lower portion of the uterine canal into the Fallopian tube by having a tampon placed in the vagina. I make it a rule never to apply a tampon in specific vaginitis.

I am extremely doubtful of the propriety of the use of tampons placed against the cervix in cases of epithelioma or cancer of the cervix uteri.

It has been suggested that we use tampons in cases of inflammation of the ovaries and the Fallopian tubes; the idea advanced is that the tampon supports the uterus and prevents the organ from dragging and exerting traction upon the uterine adnexa. The danger of using these tampons is that suppuration may take place whilst the tampon is in position, the purulent secretion will pass into the cavity of the uterus and will be prevented from passing out by the presence of the vaginal tampon, and may be forced back into the peritoneal cavity, lighting up inflammation at that point.

The treatment of inflammatory conditions of the cervix and uterine body by vaginal tampons, as suggested by Dr. Engleman, has not accomplished the good results we expected when the treatment was advised by that illustrious gynecolr zist. One reason of failure, I think, is this one point that I dwell upon with so much earnestness, that is, the discharges are prevented from flowing out of the cavity of the uterus.

In conclusion, I would advise the application of tampons only under the following conditions:

In office practice, after making an application to the endometrium, if the agent employed is likely to flow down and excoriate the external genitals, I place a very thin pledget of absorbent cotton lightly against the cervix; this will absorb the secretions; I tell my patient to remove it immediately upon her arrival home.
In cases of prolapse of the uterus, where there is no uterine catarrh, using it as a pessary. Also in cases of prolapse of the vaginal walls under similar conditions.

After plastic operations upon the vaginal walls where there is no danger of hæmorrhage taking place above the point where the tampon has been placed.

In cases of hyperplasia with little or scanty secretion from the endometrium, I frequently place a small pledget against the cervix uteri; this may be medicated with glycerine, boroglyceride, glycerate of lead, etc.

The application of tampons to correct displacements of the ante- or retro-character is very unreliable. It is so difficult to place the pledgets so as to avoid increasing the displacement. Besides, the pressure of the tampon often interferes with the circulation in the pelvis. I have frequently had patients complaining of pain located in the hip or down one leg after the application of a tampon or tampons.

\section{NON-SURGICAL TREATMENT OF CANCEROUS UTERI.}

Read in the Section of Obstetrics and Diseases of Women, at the Fortysecond Annual Meeting of the American Medical Association, held at Washington, D. C., May, I8gT.

BY C. R. REED, M.D., OF MIDDLEPORT, $O$

It is not the purpose of this paper to condemn the surgical treatment of cancer of the uterus; nor would the writer claim that a majority of the malignant diseases of that organ should be treated by medicine alone, for he admits that cancer anywhere should be treated, when practicable, by local remedies, and that general medication has little or no influence over the disease, and the vaunted specific he would only mention to condemn. The proposition to treat cancer with the curette and local applications would appear absurd after reading the above title of the paper, yet the paradox will seem logical, as we proceed to illustrate our position.

The non-surgical treatment of cancerous uteri is the disuse of the scalpel or knife, as unnecessary, in many cases; while probably a majority of cases, when first seen by the physician, require the total extirpation of the diseased organs, but it is not in the interest of medical science or suffering woman, to treat all cases of uterine cancer, at once, by what is called the radical operation. The writer pursued this course several years, telling the patient and her friends that there was nothing to be done for her but total extirpation of the cervix, at least, and better that the whole organ be removed; and as she would not follow this advice, he obstinately refused to do anything, and telling her and friends to let her die if she wished-which she usually did. He has 
since concluded that he made a mistake, that a dangerous surgical operation is not always necessary, and that his declaration and acts were not always in the interests of the patient and humanity, nor the science and art of medicine and surgery. If the suffering woman refuses to submit to the knife, of which most all of us have a dread, and is willing to undergo the more pain. ful, but less dangercus, use of the curette and the cautery, she certainly has a right to her choice, and the physician should, frequently, gracefully acquiesce. It may be claimed that this is surgical treatment. Call it minor surgery, if you please-she and her friends have a right to choose it, they taking the responsibility of the result. There are many other diseases to which women, and men also, are subject, which would best be treated by surgical operations to which they often will not submit, but they should not be abandoned to fate, if they can be cured or relieved by less objectionable means.

The prejudice against surgical operations is innate in the human race, and cannot be eradicated, and it becomes necessary to devise other means of treatment, which may often, at least, attain the same end. The woman should not be subjected to a dangerous operation if she can be cured or relieved by means less fatal. That the removal of the uterus, by any of the means recently devised, is frequently fatal, no one will deny; that cancerous uteri can be arrested in its progress, and often cured, by local treatment attended with little or no danger to life, is a well established fact. The purpose of this paper being to give the result of my own observations, I will quote but a single paragraph from authorities on the subject. In his late work on Diseases of Women, A. J. C. Skene, of Brooklyn, in speaking of treatment of cancer of the uterus, says: "A thorough curetting, followed by powerful caustics, will frequently arrest the progress of the disease."

Mrs. F., aged 6o, mother of several healthy children, had passed the menopause ten years since, and had enjoyed good health until early in I889, when she observed a red flow which at times was profuse, and rapidly destroyed her strength; she also had pelvic pain, and when the hæmorrhage was slight she had a profuse watery discharge, which had the odor characteristic of malignant disease. During i 889 she gradually lost strength and flesh, and spent most of the time in bed. She was attended alternately by two physicians, members of this Association, who had persistently kept from her the true nature of her disease. Not receiving any apparent permanent benefit from the treatment, which was both general and local, and not understanding why she did not get better, she discharged her attending physicians and sought my advice, and I first saw her February 4, I890. I found her in bed, unahle to sit up but little, emaciated, and the cancerous cachexia well marked, and to relieve her suffering she had contracted the morphine habit, with all its dire results. I should have said her parents were living, each about 90 , and she had been a widow some twenty years. On a tactile examination I found a carcinomatous cervix, patulous, and that characteristic feel as when you poke your finger into the nozzle of a shotgun. There were also several nodules which felt like large shot in the posterior part of the vagina. The examination caused hæmorrhage, and on expanding the blades of a bivalve speculum, it was so profuse that I felt relieved when I secured its arrest by applying the pure persulphate of iron.

She was told the nature of her disease, the prognosis of such cases, and was advised to undergo the removal of the diseased organ. This she firmly and pleasantly declined, believing she would not live through the operation, and I did not urge it, as I was of the same opinion. She implored me to do what I could to prolong her life; she wanted to live until her parents passed away, and asked that the attacks of hæmorrhage which she so much dreaded be arrested. I did not think that the body of the uterus or its appendages were involved, and had soine hopes that temporary relief might result from active local treatment. She lived in an adjoining State, and I visited her once a week, and each time used the sharp curette, followed by the pure chromic acid. I prepared a swab of absorbent cotton, as large as would pass into the cervical canal after I had rapidly reamed it out with the curette, and on withdrawing the latter, immediately dipped the swab into water, then into the dry chromic acid, and carried it as far as possible into the cervix and cavity, and held it there until the hissing and smoke ceased, then withdrew the swab, packed the cervix with cotton saturated with the pure persulphate, which was directed to be removed by a string attached for that purpose, in three or four days. The hamorrhage from the use of the curette would be fearful, but immediately stopped on contact of the chromic acid. She was directed to let me know should hæmorrhage occur in the intervals of my visits, and but once did she send for me.

When I left home a year ago to attend the meeting of this Association at Nashville, I arranged with a physician near her to apply the treatment in my absence. This was continued regularly for several months, the hæmorrbage ceasing, the local- symrtoms disappearing, the general health improving, flesh, strength and color returning, and when I left home in October last for a several weeks' visit in the West, I had no forebodings in regard to her case, and when I saw her in November I found her doing her work, apparently well and happy, with scarcely a trace 
of her former disease on examination. I have intra-uterine syringe, when ten minims of a ro not seen her for several months, though frequent- per cent. solution is inserted into the uterine cav$1 \mathrm{y}$ in sight of her house, and learn that she is ap- ity. Continue at once with the dilating of the parently well. I commenced the treatment of cervix until you have it completed to the extent this case with a mental prognosis that it would desired, and then thoroughly curette the entire end fatally in from four to six months-but dur- uterine cavity. This can be done with a slight ing a professional life of nearly forty years I have attended no case that the treatment gave me more satisfaction than this, and I have the still greater pleasure of the assurance that the treatment at no time and in no way imperiled her life.

Will the same treatment be generally successful in similar cases? This can only be determined on trial. Could the treatment be as well carried out during menstrual life? Probably not. Will the apparent cure in this case be permanent? Of this I will not affirm, neither can it be foretold of the cases in which the radical operation of entire removal has been done.

It would not be consistent with the intended limits of this paper to give other cases in detail, and had no other cases come under my observation with a similar result, and no case in the literature on the subject, this alone would be sufficient to induce me to use the same or similar treatment before resorting to the operation of removal of the entire organ and appendages, with the present mortality attending the radical operation.

\section{ON THE USES OF COCAINE IN GYNE- COLOGICAL SURGERY.}

Read in the Section of Obstetrics and Diseases of Women, at the Forly-second Annual Meeting of the American Medical Association, held at IV'ashington, D.C., May, 1891 .

BY WM. H. HUMISTON, M.D., OF CLEVELAND, OHIO.

I have had an experience in the use of cocaine in gynecological operations during the past three years that has been eminently satisfactory, and I purpose in as few words as possible to lay the method and my experience before you.

I will first describe the manner of its use in dilating and curetting. Ten minutes before using the cocaine I give a tablespoonful of whisky or brandy. Place patient in the left lateral position, and with Sim's speculum expose the cervix-steady the uterus with a tenaculum inserted in anterior lip. Then take a bypodermic syringe with a fiue needle, filled with a 4 per cent. solution of cocaine, which has two minims of pure phenol in each half-ounce of solution, and inject five minims into the posterior lipwait two minutes, then secure a firm hold with bullet forceps, which will be painless. Proceed and inject in several portions of the cervical canal an amount equal to about twenty minims. I now commence my dilation, with the graduated hard rubber dilators until the cervix is sufficiently dilated to permit the entrance of the degree of pain, and in many cases with none at all. I have not given an anæsthetic, other than just described, in dilating or curetting, during the past three years, and the cases include many primiparæ.

For the operation of trachelorrhaphy you can use cocaine and have absolutely a painless operation. Inject the angle, and the sides that you desire to freshen, or a wedge-shaped piece that must come away, and you can complete it all without the patient making a complaint. You will rarely require to use over one-half drachm of a 4 per cent. solution.

For restoration of the perineum in the majority of cases I adopt the split-flap method, and with one puncture of the hypodermic needle in the median line I anæsthetize the whole field of operation, and use only from thirty to forty minims of a 4 per cent. solution.

The hour for operating is usually $90^{\prime}$ clock in the morning. The patient omits breakfast, and ten or fifteen minutes before the operation the alcoholic stimulant is given.

I say to the nervous timid patients, who are afraid that without chloroform, or ether, that their suffering will be great, that I will stop at any time that they say that they are suffering pain, and give them chloroform. This secures their confidence, allays their fears, and I have yet to hear the first request for another form of anæsthetic.

With this method we avoid the frequent and prolonged nausea and vomiting that occurs after the administration of chloroform or ether. The patients, with but few exceptions, relish their dinner, and their progress towards recovery is repid and satisfactory. Had chloroform been given, and been followed with persistant vomiting, that so frequently occurs, the whole success of the operation, especially if it be for the restoration of the perineum, would be in jeopardy.

Quite frequently I find patients who are very sensitive, and very small doses will induce short and sighing respiration, together with peculiar sensations in præcordia, accompanied by great anxiety. Stimulants, promptly administered, control this very quickly and all unfavorable symptoms subside.

Twice I have dilated the urethra for fissure and irritable caruncula with but slight suffering, and a satisfactory result was obtained.

I assisted in one case of Alexander's operation where cocaine was used, but two grains in all, one grain injected on each side at intervals of onehalf hour. While this was not painless the pa- 